\title{
Nanoparticles in Sensing Applications: On What Timescale Do Analyte Species Adsorb on the Particle Surface?
}

\author{
Enno Kätelhön and Richard G. Compton* \\ Physical and Theoretical Chemistry Laboratory, South Parks Road, \\ Oxford, OX1 3QZ, England / United Kingdom. \\ ${ }^{*}$ Corresponding author. Tel: $+44(0) 1865$ 27595\%. E-mail: \\ richard.compton@chem.ox.ac.uk
}

\begin{abstract}
The recent decade saw much interest in sensors based on nanoparticles. Such sensors typically employ sensing mechanisms that utilise the adsorption of analyte species on the nanoparticle surfaces, while adsorption induces changes in the physical properties of the nanoparticles. In this work, we introduce an analytical model for the rate of adsorption of analyte species on the nanoparticle surface. Expressions for the fractional surface coverage and the number of adsorbed molecules as a function of time are derived assuming spherical nanoparticles. Moreover, we provide values for common experimental conditions and show that for small nanoparticles $(r<10 \mathrm{~nm})$ a surface coverages of $30 \%$ can be reached in less than $1 \mathrm{~s}$ at adsorbent concentrations as low as $50 \mathrm{nM}$.
\end{abstract}

\section{Introduction}

Research on nanoparticles has received vast attention during recent years. Due to their special physiochemical properties, nanoparticles are of interest in fundamental research and simultaneously open up a wide range of novel sensing mechanisms, while potential applications cover different sensing fields that range from biosensors ${ }^{1 ; 2}$ to the developement of enviromental sensors. ${ }^{3}$ One of the main advantages of nanoparticle-based sensing over other approaches arises from the nanoparticles' high surface to volume ratio, which provides a comparably large surface area available for reaction within a small sample volume. Furthermore, accelerated mass transport of analyte species to the nanoparticle surface is enabled through spherical diffusion and can significantly reduce the time that is required to perform a measurement as we will discuss fully below. 
In a typical sensing application, nanoparticles are immersed into the sample solution, enabling the direct interaction between analyte species and the nanoparticle surface, for instance via physical adsorption or chemical reaction. These interactions may impact the physical characteristics of the nanoparticles, which can then be measured via different methods as specified by Saha et al.: ${ }^{4}$ Employed approaches include colorimetric sensing, ${ }^{5-8}$ fluorescence-based sensing, ${ }^{9}$ electrical and electrochemical sensing, ${ }^{2 ; 10 ; 11}$ surface plasmon resonance sensors, ${ }^{12 ; 13}$ surface enhanced Raman scattering (SERS)-based sensing, ${ }^{14 ; 15}$ and quartz crystal microbalance-based sensing. ${ }^{16}$

In this study, we introduce an analytical model for the description of adsorption processes on the surface of freely diffusing nanoparticles and derive expressions for the fractional coverage of nanoparticles with adsorbent molecules and the number of adsorbed molecules as a function of time. We further calculate values for typical experimental settings, which may be used as a reference for experimentalists.

\section{Theory}

\section{$2.1 \quad$ Theoretical model}

We consider a single spherical nanoparticle that freely diffuses in a solution containing adsorbing molecules at a given concentration $c^{*}$. These molecules adsorb immediately and permanently whenever they touch the nanoparticle surface and feature a diffusion coefficient $D$ that is significantly higher than the diffusion coefficient $D_{n p}$ of the nanoparticle $\left(D \gg D_{n p}\right)$. Once adsorbed, each molecule occupies the surface area $A_{b}^{m}$ on the nanoparticle surface

$$
A_{b}^{m}=\pi r_{b}^{2}
$$

where $r_{b}$ is the radius of the adsorbing molecules, and fully blocks this area for the adsorption of other molecules. At the beginning of the experiment, the adsorbing molecules are equally distributed in solution and the nanoparticle surface is free from adsorbing molecules. A graphical illustration of our model can be found in Figure 1.

Since our model does not consider the diffusive movement of the nanoparticle in regard to the surrounding solution, our calculations give lower estimates for the adsorption rate. The thermal motion of the nanoparticle will therefore enhance the rate of adsorption in the experiment.

We presume that results are widely unaffected by variations in the particle shape, since molecules adsorb immediately and permanently on the nanoparticle surface. This assumption is supported by a previous studiy, in which we investigated the chronoamperometric response of differently shaped nanoparticles on a supporting surface in the diffusion limited case, and found that different shapes may lead to equal chronograms. ${ }^{17}$ 


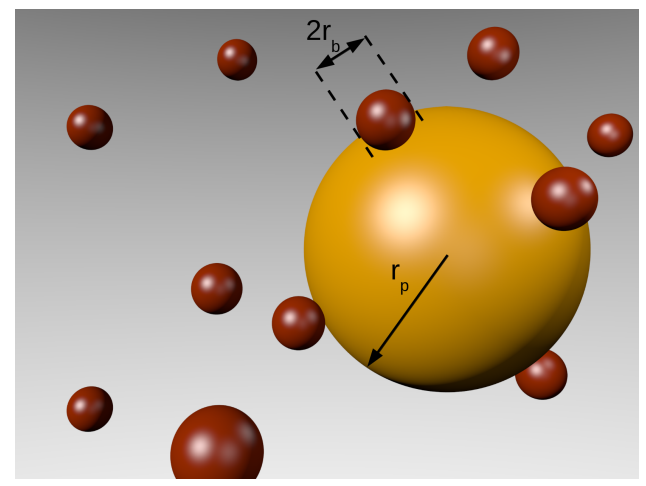

Figure 1: Graphical illustration of the model used in this study.

\subsection{Fractional surface coverage}

In this section, we derive an expression for the fractional surface coverage $\theta$ of the nanoparticle with adsorbing molecules as a function of time. The presented derivation is based on a previously published study focussing on macroelectrodes. ${ }^{18}$ Here, we modify this approach for the case of a spherical nanoparticle in solution. In order to improve readability, an overview of all used variables is given in Table 1 .

We first define the unoccupied surface area $A(t)$ of the nanoparticle as a function of $\theta(t)$

$$
A(t)=(1-\theta(t)) A_{0}
$$

where $A_{0}$ is the overall surface area of the nanoparticle and $\theta(t)$ the fractional surface coverage, which is a function of time and may adopt values between 0 and 1 for the limiting cases of a free and a fully blocked surface, respectively.

The fractional surface coverage $\theta(t)$ can further be written as a function of the number of adsorbed molecules $n_{a d s}(t)$

$$
\theta(t)=\frac{A_{b}(t)}{A_{0}}=\frac{n_{a d s}(t) A_{b}^{m}}{A_{0}}
$$

where $A_{b}(t)$ is the total blocked surface area and $A_{b}^{m}$ is the surface area that is blocked by a single adsorbed molecule. Since $n_{a d s}$ is an integer, $\theta(t)$ is quantised to multiples of $A_{b}^{m} / A_{0}$, which is negligibly small for most applications. Hence, we treat $\theta(t)$ as a steady function in the following derivation.

In case of a spherical surface, the flux of analyte molecules to the surface can be written in analogy to Cottrell equation ${ }^{19}$ for spherical electrodes.

$$
\frac{d n}{d t}=N_{A} A_{0} D c^{*}\left(\frac{1}{\sqrt{\pi D t}}+\frac{1}{r_{p}}\right)
$$

where $d n / d t$ is the flux of analyte molecules and $c^{*}$ is the concentration of analyte species present in solution. 


\begin{tabular}{|l|l|}
\hline Variable & Description \\
\hline \hline$\theta(t)$ & $\begin{array}{l}\text { Fractional surface coverage of } \\
\text { the nanoparticle at the time } t\end{array}$ \\
\hline$A(t)$ & $\begin{array}{l}\text { Total unoccupied surface area } \\
\text { of the nanoparticle at the time } \\
t\end{array}$ \\
\hline$A_{0}$ & $\begin{array}{l}\text { Total surface area of the } \\
\text { nanoparticle }\end{array}$ \\
\hline$A_{b}(t)$ & $\begin{array}{l}\text { Total blocked surface area of } \\
\text { the nanoparticle at the time } t\end{array}$ \\
\hline$n_{a d s}(t)$ & $\begin{array}{l}\text { Total number of adsorbed } \\
\text { molecules on the nanoparticle } \\
\text { surface at the time } t\end{array}$ \\
\hline$A_{b}^{m}$ & $\begin{array}{l}\text { Surface area blocked by a sin- } \\
\text { gle adsorbed molecule }\end{array}$ \\
\hline$\frac{d n}{d t}$ & $\begin{array}{l}\text { Flux of molecules to the par- } \\
\text { ticle surface }\end{array}$ \\
\hline$r_{b}$ & $\begin{array}{l}\text { Radius of the adsorbing } \\
\text { molecules }\end{array}$ \\
\hline$D$ & $\begin{array}{l}\text { Diffusion coefficient of the ad- } \\
\text { sorbing molecules }\end{array}$ \\
\hline$c^{*}$ & $\begin{array}{l}\text { Bulk concentration of adsorb- } \\
\text { ing molecules in solution } \\
\text { Radius of the nanoparticle }\end{array}$ \\
\hline$r_{p}$ & \\
\hline
\end{tabular}

Table 1: Overview of all variables employed in this study.

We can use this equation to derive an expression for the number of adsorbed molecules, $n_{a d s}(t)$. Since molecules adsorb immediately whenever they touch a nanoparticle, the rate of adsorbing molecules mathematically equals the flux of analyte molecules to the surface. Furthermore, we replace $A_{0}$ with $A(t)$, which acts as a good approximation for the case of a low surface coverage. Considering that due to its thermal energy the nanoparticle moves and rotates at room temperatures, we presume that this approximation even holds for higher values of $\theta(t) . \quad n_{a d s}(t)$ can then be written as

$$
\frac{d n_{a d s}(t)}{d t}=N_{A} A(t) D c^{*}\left(\frac{1}{\sqrt{\pi D t}}+\frac{1}{r_{p}}\right)
$$

If we now combine the equations (1), (2), and (5), we obtain a differential equation for $\theta(t)$

$$
\frac{d \theta}{d t}=\pi r_{b}^{2} N_{A}(1-\theta) D c^{*}\left(\frac{1}{\sqrt{\pi D t}}+\frac{1}{r_{p}}\right)
$$


This equation can be solved via the separation of the variables $\theta$ and $t$. Rearrangement of the variables and integration leads to

$$
\int d \theta \frac{1}{1-\theta}=\pi r_{b}^{2} N_{A} D c^{*} \int d t\left(\frac{1}{\sqrt{\pi D t}}+\frac{1}{r_{p}}\right)
$$

We can solve the integrals on both sides independently and obtain

$$
\ln (1-\theta)=\pi r_{b}^{2} N_{A} D c^{*}\left(\frac{2 \sqrt{t}}{\sqrt{\pi D}}+\frac{t}{r_{p}}\right)+\text { const. }
$$

Since the nanoparticle surface is free from adsorbing molecules at the beginning of the experiment, we can use the boundary condition $\theta(t=0)=0$ to determine the integration constant. By this means we find the desired expression for the fractional surface coverage $\theta(t)$ as a function of time

$$
\theta(t)=1-\exp \left(-\pi r_{b}^{2} N_{A} D c^{*}\left(\frac{2 \sqrt{t}}{\sqrt{\pi D}}+\frac{t}{r_{p}}\right)\right)
$$

Using Equation (3), we can transform this equation into an expression for the number of adsorbed molecules as a function of time

$$
\begin{gathered}
n_{a d s}(t)=\frac{A_{0}}{A_{b}^{m}} \theta(t) \\
=\frac{5 r_{p}^{2}}{8 r_{b}^{2}}\left(1-\exp \left(-\pi r_{b}^{2} N_{A} D c^{*}\left(\frac{2 \sqrt{t}}{\sqrt{\pi D}}+\frac{t}{r_{p}}\right)\right)\right)
\end{gathered}
$$

which is here given as a steady function since above we treat $\theta(t)$ as a positive real number $\left(\theta(t) \in \mathbb{R}^{+}\right)$.

\subsection{Fractional surface coverage in the diffusional steady state}

Due to the small size of nanoparticles, the diffusion field around a nanoparticle quickly reaches a steady state as soon as the nanoparticle is exposed to adsorbing molecules in the surrounding solution. Since the time that is required to reach this state is typically of the order of a few $100 \mathrm{~ns}$ for particles featuring a diameter below $20 \mathrm{~nm},{ }^{17}$ we can approximate $\theta(t)$ in Equation (9) by using the assumption that the system instantaneously reaches the diffusional steady state in the derivation of $\theta(t)$. For this reason, we first determine the steady-state flux to a spherical electrode: we calculate the flux of molecules to the surface for the long-term limit $(t \rightarrow \infty)$ and find that the time-dependent term $(\pi D t)^{-0.5}$ vanishes in Equation (4). Hence, we can simplify the flux to

$$
\frac{d n}{d t}=\frac{N_{A} A_{0} D c^{*}}{r_{p}}
$$


Now we can calculate the fractional surface coverage in analogy to the derivation in Section 2.2 and find that $\theta_{s s}(t)$ can be expressed through

$$
\theta_{s s}(t)=1-\exp \left(-\frac{\pi r_{b}^{2} N_{A} D c^{*} t}{r_{p}}\right)
$$

which equals $\theta(t)$ in Equation (9), if the dependency on $\sqrt{t}$ is neglected. The number of adsorbed molecules as a function of time is then given by

$$
\begin{gathered}
n_{a d s, s s}(t)=\frac{A_{0}}{A_{b}^{m}} \theta_{s s}(t) \\
=\frac{5 r_{p}^{2}}{8 r_{b}^{2}}\left(1-\exp \left(-\frac{\pi r_{b}^{2} N_{A} D c^{*} t}{r_{p}}\right)\right)
\end{gathered}
$$

For most applications, this result approximates $\theta(t)$ well and can be used equivalently. The comparison between the two time-dependent terms in our result for $\theta(t)$ in Equation (9) shows that the contribution of the time-dependent term in the flux $(\propto \sqrt{t}$, Equation $(4))$ to $\theta(t)$ is significantly smaller than the contribution of the steady-state term $(\propto t)$. If we calculate the two factors $C_{1}$ and $C_{2}$

$$
C_{1} \cdot \sqrt{t}:=\frac{2 \sqrt{t}}{\sqrt{\pi D}} \wedge C_{2} \cdot t:=\frac{t}{r_{p}}
$$

for typical experimental values, $D=10^{-9} \mathrm{~m}^{2} \frac{1}{s}$ and $r_{p}=10 \mathrm{~nm}$, we find that the value of $C_{2}$ exceeds $C_{1}$ by four orders of magnitude.

\section{Results and discussion}

We calculate the fractional coverage $\theta(t)$ of nanoparticles that feature sizes between 10 and $50 \mathrm{~nm}$ and are exposed to analyte concentrations ranging from $50 \mathrm{nM}$ to $2.5 \mu \mathrm{M}$. For our calculation we use the diffusion coefficients of acetone $\left(D=1.16 \cdot 10^{-9} \mathrm{~m}^{2} / \mathrm{s}\right),{ }^{20}$ glutathione $\left(D=6.7 \cdot 10^{-10} \mathrm{~m}^{2} / \mathrm{s}\right),{ }^{21}$ and a large macromolecule $\left(D=5 \cdot 10^{-11} \mathrm{~m}^{2} / \mathrm{s}\right)$. The radius of the blocking molecule is herein approximated through the respective molecular hydrodynamic radius and calculated via the Stokes-Einstein-Equation, which can be obtained by combining Einstein-Smoluchowski equation ${ }^{22 ; 23}$ and Stokes equation

$$
r_{b}=\frac{k_{B} T}{6 \pi \eta D}
$$

where $k_{B}$ represents the Boltzmann constant, $T$ the room temperature, and $\eta$ the viscosity of water. The graphs for $\theta(t)$ are computed on the basis of Equation (9) and can be found in Figure 2 and 3 for the diffusion coefficients of acetone and glutathione, respectively. 

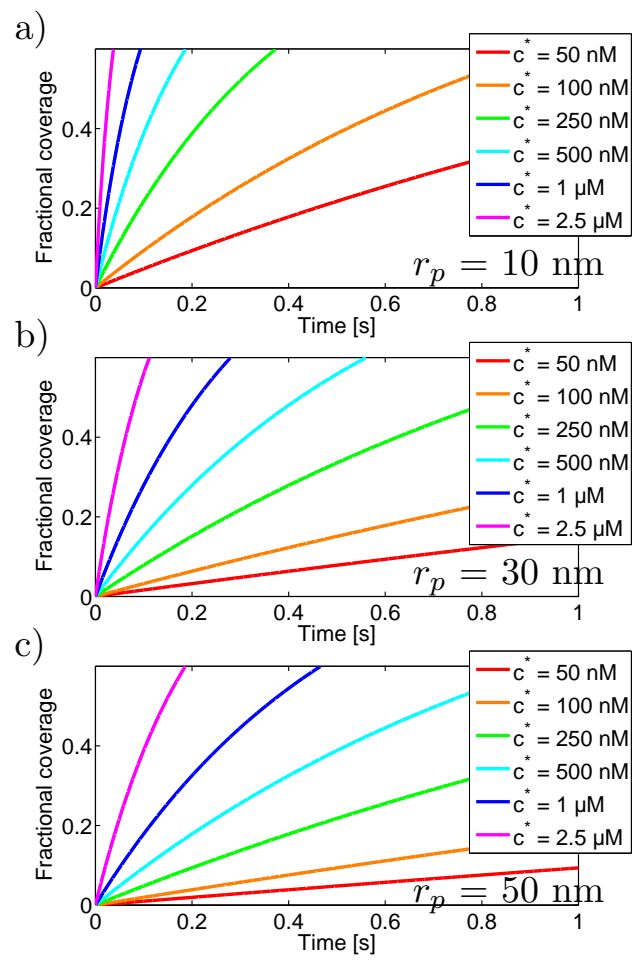

Figure 2: Fractional coverage of a nanoparticle surface as a function of time. The radius $r_{p}$ of the nanoparticle is varied between 10 and $50 \mathrm{~nm}(2 \mathrm{a}$ to 2 c) while the employed diffusion coefficient $D=1.16 \cdot 10^{-9} \mathrm{~m}^{2} / \mathrm{s}$ equals the diffusion coefficient of acetone. ${ }^{20}$ The blocking molecule's radius, $r_{b}$, is calculated via the Stokes-Einstein equation and amounts to $0.19 \mathrm{~nm}$.

In both Figures, we can find that the temporal evolution of the fractional coverage strongly depends on the nanoparticle size as well as the initial concentration of adsorbing nanoparticles, while smaller sizes and higher adsorbent concentrations lead to an accelerated increase in fractional surface coverage. In case of both adsorbing molecules, fractional surface coverages of more than $30 \%$ are reached within less than $1 \mathrm{~s}$ for a $10 \mathrm{~nm}$ nanoparticle.

Figure 4 presents the number of adsorbed molecules as function of time, while results are calculated through Equation (10). For direct comparability, here, we first again employ the values of the acetone molecule for the adsorbing species.

The presented data demonstrates a strong dependency of the number of adsorbed molecules on the nanoparticle size and the concentration of adsorbing molecules in solution. Larger radii and higher concentrations result in an increase in the rate of adsorbing molecules. At concentrations as low as $50 \mathrm{nM}$, more than 100 molecules adsorb in less than $30 \mathrm{~ms}$ in case of a $50 \mathrm{~nm}$ nanoparticle (4b).

Since absolute numbers of adsorbing species typically have more rele- 

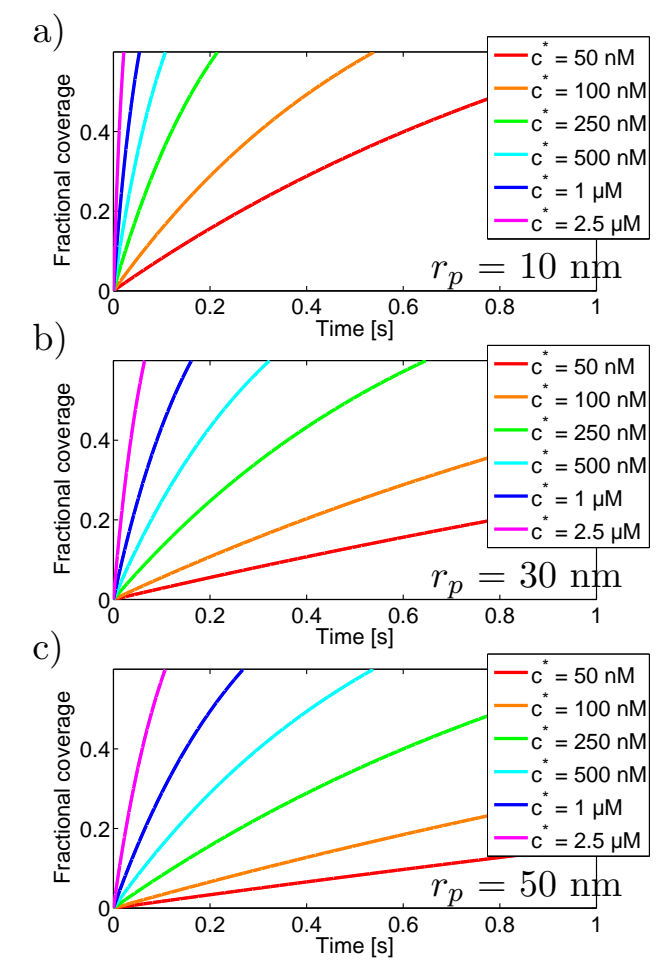

Figure 3: Fractional coverage of a nanoparticle surface in the case of adsorbing glutathione molecules. The diffusion coefficient $D$ is set to $6.7 \cdot 10^{-10}$ $\mathrm{m}^{2} / \mathrm{s},{ }^{21}$ which corresponds to a Stokes radius $r_{b}$ of $0.34 \mathrm{~nm}$.

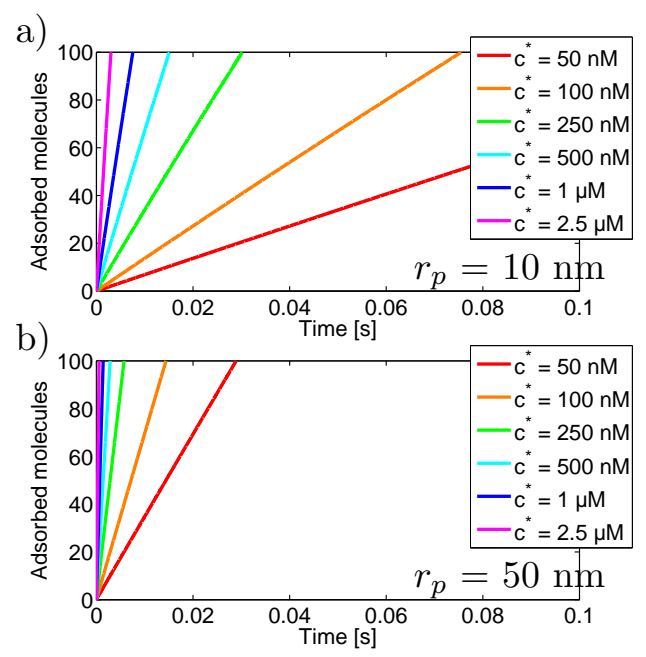

Figure 4: Number of adsorbed molecules on the nanoparticle surface as a function of time. We employ the parameters specified in Figure 2.

vance in the case of adsorbing biological macromolecules, we additionally present data for a diffusion coefficient of $5 \cdot 10^{-11} \mathrm{~m}^{s} / \mathrm{s}$ in Figure 5 . For a 


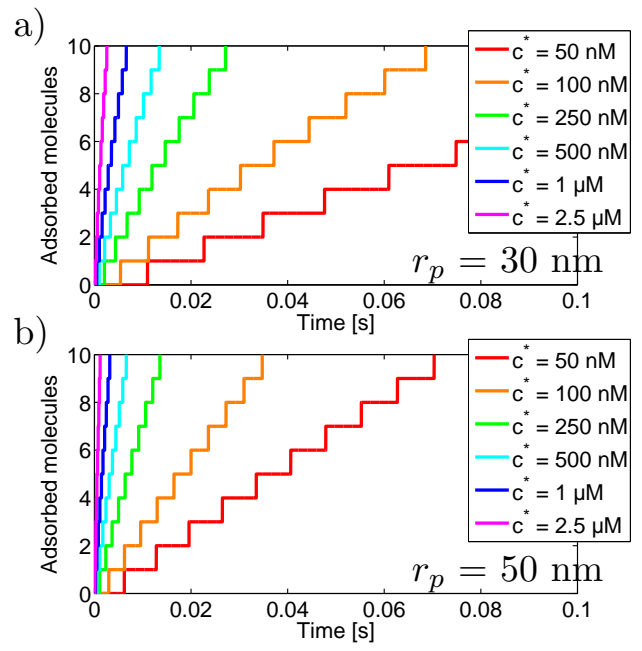

Figure 5: Number of adsorbed macromolecules on the nanoparticle surface. Data is calculated for a diffusion coefficient of $5 \cdot 10^{-11} \mathrm{~m}^{2} / \mathrm{s}$ and a corresponding Stokes radius of $\mathrm{r}_{b}=4.5 \mathrm{~nm}$.

clearer demonstration we use the floor function to calculate the number of adsorbed macromolecules

$$
n_{\text {ads }}(t)=\left\lfloor n_{a d s}(t)\right\rfloor
$$

where the floor function $\lfloor\ldots\rfloor$ maps $n_{a d s}(t)$, which we treated as a positive real number in our derivation $\left(n_{\text {ads }}(t) \in \mathbb{R}^{+}\right)$, to the value of the closest lower integer. Since each adsorbed molecule blocks a comparably large fraction of the surface area, only few molecules can adsorb and the adsorption of single molecules is seen in the graph. Hence, the graph exhibits a digital response, in which each step corresponds to a single adsorbing molecule.

We find that the rate of adsorbing molecules is significantly slower in the case of the macromolecule than with the previously discussed molecules, which is due to the lower diffusion coefficient. In case of a $50 \mathrm{~nm}$ particle, 10 macromolecules can adsorb within $80 \mathrm{~ms}$ at all considered concentrations (5b), whereas the rate decreases with the nanoparticle size (5a).

\section{Conclusions}

We introduce an analytical model for the description of the adsorption of analyte species on the surface of freely diffusing nanoparticles and derive time-dependent terms for the fractional surface coverage of the nanoparticle and the total number of adsorbed molecules. Furthermore, we calculate values for typical experimental settings, which may serve as a helpful reference in the development of novel sensors that are based on adsorption to 
nanoparticles. The presented analytical model can hence be used to estimate the response time of such sensors and can be employed as a tool to validate experimental results by comparison of the measured response time to the theoretical result.

\section{Acknowledgments}

The research leading to these results has received partial funding from the European Research Council under the European Union's Seventh Framework Programme (FP/2007-2013) / ERC Grand Agreement n. [320403]. We thank Eden Tanner for proofreading the manuscript.

\section{Keywords}

- Nanoelectrochemistry

- Nanoparticles

- Adsorption

- Biosensors

\section{References}

[1] P. Alivisatos, Nat. Biotech., 2004, 22, 47-52.

[2] X. Luo, A. Morrin, A. J. Killard and M. R. Smyth, Electroanal., 2006, 18, 319-326.

[3] L. Wang, W. Ma, L. Xu, W. Chen, Y. Zhu, C. Xu and N. A. Kotov, Mat. Sci. Eng. R., 2010, 70, 265-274.

[4] K. Saha, S. S. Agasti, C. Kim, X. Li and V. M. Rotello, Chem. Rev., 2012, 112, 2739-2779.

[5] R. Elghanian, J. Storhoff, R. Mucic, R. Letsinger and C. Mirkin, Science, 1997, 277, 1078-1081.

[6] J. Storhoff, R. Elghanian, R. Mucic, C. Mirkin and R. Letsinger, J. Am. Chem. Soc., 1998, 120, 1959-1964.

[7] J. Storhoff, A. Lazarides, R. Mucic, C. Mirkin, R. Letsinger and G. Schatz, J. Am. Chem. Soc., 2000, 122, 4640-4650.

[8] J. Liu and Y. Lu, J. Am. Chem. Soc., 2003, 125, 6642-6643.

[9] T. Taton, C. Mirkin and R. Letsinger, Science, 2000, 289, 1757-1760. 
[10] R. Polsky, R. Gill, L. Kaganovsky and I. Willner, Anal. Chem., 2006, 78, 2268-2271.

[11] A. Merkoçi, FEBS Journal, 2007, 274, 310-316.

[12] C. Haynes and R. Van Duyne, J. Phys. Chem. B, 2001, 105, 5599-5611.

[13] J. N. Anker, W. P. Hall, O. Lyandres, N. C. Shah, J. Zhao and R. P. Van Duyne, Nat. Mater., 2008, 7, 442-453.

[14] S. Nie and S. Emory, Science, 1997, 275, 1102-1106.

[15] G. Goddard, L. O. Brown, R. Habbersett, C. I. Brady, J. C. Martin, S. W. Graves, J. P. Freyer and S. K. Doorn, J. Am. Chem. Soc., 2010, 132, 6081-6090.

[16] X. Mao, L. Yang, X.-L. Su and Y. Li, Biosens. and Bioelectron., 2006, 21, 1178-1185.

[17] E. Kätelhön, E. O. Barnes, K. J. Krause, B. Wolfrum and R. G. Compton, Chem. Phys. Lett., 2014, 595-596, 31-34.

[18] E. Kätelhön, W. Cheng, C. Batchelor-McAuley, K. Tschulik and R. G. Compton, accepted for publication in ChemElectroChem, 2014.

[19] A. J. Bard and L. Faulkner, Electrochemical Methods: Fundamentals and Applications (Second Edition), John Wiley and Sons, Inc., 2001.

[20] E. L. Cussler, Diffusion: Mass Transfer in Fluid Systems, Cambridge University Press, 1997.

[21] W. Jin and H. Chen, Chromatographia, 2000, 52, 17-22.

[22] A. Einstein, Ann. Phys., 1905, 322, 549-560.

[23] M. von Smoluchowski, Ann. Phys., 1906, 326, 756-780. 